\title{
ANALYTIC MAPS BETWEEN TORI ${ }^{1}$
}

\author{
BY HEINZ G. HELFENSTEIN
}

Communicated by F. W. Gehring, February 20, 1969

We obtain the conditions under which nonconstant complex analytic maps between two tori exist, as well as the set of all such maps. The arguments carry over to analytic maps between higher-dimensional tori, as well as other categories of maps; e.g. affine maps between flat manifolds. Since the precise statements of the results become complicated, even under simple circumstances, we restrict ourselves to the case of 2-dimensional tori. The main point of interest lies in the existence of mutually disjoint dense subsets of the manifold of all tori, which yield different sets of analytic homotopy classes of maps. Details will appear elsewhere.

Let $\mathrm{GL}^{+}(2, Q)$ denote the group of all $2 \times 2$ matrices with real rational entries and positive determinant; $\operatorname{SL}(2, Z)$ the subgroup of all $2 \times 2$ matrices with real integer entries and determinant +1 . Then the groups

$$
G=\mathrm{GL}^{+}(2, Q) /\{\lambda I: \lambda \neq 0, \text { rational }\}
$$

with

$$
I=\left(\begin{array}{ll}
1, & 0 \\
0, & 1
\end{array}\right)
$$

and

$$
F=\operatorname{SL}(2, Z) /\{ \pm I\}
$$

act effectively on

$$
H=\{z \in C: g z>0\}
$$

by letting

$$
g(z)=\frac{a z+b}{c z+d}
$$

for

$$
g=\left(\begin{array}{ll}
a, & b \\
c, & d
\end{array}\right) \in \mathrm{GL}^{+}(2, Q)
$$

${ }^{1}$ Work supported by the National Research Council of Canada. 
The isometry classes of 2-dimensional tori provided with a Riemannian metric of constant vanishing curvature are parametrized by $R^{+} \times(H / F) .\left(R^{+}=\right.$positive reals). A point $\left(r^{2}, \tau\right) \in R^{+} \times(H / F)$ represents the class of $E^{2} / \Gamma$, where $\Gamma$ is the group of Euclidean motions generated by the two translations

$$
\begin{gathered}
t_{1}(z)=z+r \text { and } t_{2}(z)=z+r h, \\
h \in \tau=F(h) \subset H .
\end{gathered}
$$

Definition. A torus $\left(r^{2}, \tau\right)$ is called ample if there exists $h \in \tau$ with R $h$ and $|h|^{2}$ both rational numbers.

THEOREM 1. Two tori with $\tau_{1}, \tau_{2} \in H / F$ admit a nonconstant analytic map if and only if there exist $h_{i} \in \tau_{i}, T \in G$, with $h_{1}=T\left(h_{2}\right)$. [We write then $\tau_{1} \sim \tau_{2}$.] Hence the manifold $H / F$ is partitioned into dense subsets of orbits under $G$.

For two tori $\left(\gamma_{i}^{2}, \tau_{i}\right), i=1,2$, with $\tau_{1} \sim \tau_{2}$ there exists an analytic covering map $\tau_{1} \rightarrow \tau_{2}$ with a smallest number $a$ of sheets. (The determination of $a$ can be reduced to a manipulation of quadratic forms depending on $\tau_{1}$ and $\tau_{2}$.) We have then

THEOREM 2. Let $T_{i}=\left(r_{i}^{2}, \tau_{i}\right), i=1,2$, be two tori with $\tau_{1} \sim \tau_{2}$, both nonample. Then the analytic maps $T_{1} \rightarrow T_{2}$ are given by their lifts to the universal covering planes as

$$
F_{\kappa, D}(z)=\left(r_{2} / r_{1}\right) \kappa z+D,
$$

where $\kappa$ runs through all integers and $D$ through all complex constants.

Theorem 3. Let $T_{i}=\left(\gamma_{i}^{2}, \tau_{i}\right), i=1,2$ be ample tori with $\tau_{1} \sim \tau_{2}$. Then there exist complex numbers $h_{i} \in \tau_{i}$ with $h_{1}=a h_{2}$.

Let

$$
2 \Re h_{2}=p / q, \quad\left|h_{2}\right|^{2}=r / s,
$$

$q>0, r>0, s>0$ integers, g.c.d. $(p, q)=$ g.c.d. $(r, s)=1$,

$$
\begin{aligned}
& \text { g.c.d. }(q, s)=g, \quad q^{\prime}=q / g, \quad s^{\prime}=s / g, \\
& g^{\prime}=\text { g.c.d. }(a, q), \quad a^{\prime}=a / g^{\prime}, \quad q^{\prime \prime}=q / g^{\prime}, \\
& g^{\prime \prime}=\text { g.c.d. }\left(a^{\prime}, s^{\prime}\right), \quad a^{\prime \prime}=a^{\prime} / g^{\prime \prime}, \quad s^{\prime \prime}=s^{\prime} / g^{\prime \prime}, \\
& A_{1}=r_{2} / r_{1}, \quad A_{2}=\left(r_{2} / r_{1}\right) q^{\prime \prime} s^{\prime \prime} h_{2} \text {. }
\end{aligned}
$$

Then the lifts of all analytic maps $T_{1} \rightarrow T_{2}$ have the form $F_{k_{1}, \kappa_{2}, D}(z)$ $=\left(\kappa_{1} A_{1}+\kappa_{2} A_{2}\right) z+D$, with arbitrary integers $\kappa_{1}, \kappa_{2}$ and arbitrary complex constants $D$. 
1969]

ANALYTIC MAPS BETWEEN TORI

859

THEOREM 4. With the above notations the Riemann-Hurwitz relation is generalized as follows: The number of sheets is $N=\kappa^{2} a$ in the case of Theorem 2;

$$
N=a^{\prime \prime}\left(g^{\prime} g^{\prime \prime} \kappa_{1}^{2}+p g^{\prime \prime} s^{\prime \prime} \kappa_{1} \kappa_{2}+r q^{\prime} q^{\prime \prime} s^{\prime \prime} \kappa_{2}^{2}\right)
$$

in the case of Theorem 3.

University of Ottawa, Ottawa, Canada 